\title{
What clues are available for differential diagnosis of headaches in emergency settings?
}

\author{
Ertan Mert · Aynur Özge $\cdot$ Bahar Taşdelen $\cdot$ \\ Arda Yılmaz $\cdot$ Nursel G. Bilgin
}

Received: 2 October 2007/ Accepted: 9 January 2008/Published online: 5 February 2008

(C) Springer-Verlag 2008

\begin{abstract}
The correct diagnosis of headache disorders in an emergency room is important for developing early management strategies and determining optimal emergency room activities. This prospective clinical based study was performed in order to determine demographic and clinical clues for differential diagnosis of primary and secondary headache disorders and also to obtain a classification plot for the emergency room practitioners. This study included 174 patients older than 15 years of age presenting in the emergency room with a chief complaint of headache. Definite headache diagnoses were made according to ICHD-II criteria. Classification and regression tree was used as new method for the statistical analysis of the differential diagnostic process. Our 174 patients with headache were diagnosed as basically primary $(72.9 \%)$ and secondary $(27.1 \%)$ headaches. Univariate analysis with cross tabs showed three important results. First, unilateral pain location caused 1.431-fold increase in the primary headache risk
\end{abstract}

This study was presented as an oral presentation at the 42nd Congress of Turkish Neurological Society in Antalya, 2006.

E. Mert · A. Özge $\cdot$ B. Taşdelen · A. Yılmaz $\cdot$ N. G. Bilgin Mersin University School of Medicine, Mersin, Turkey

e-mail: drertanmert@yahoo.com

B. Taşdelen

e-mail: bahartasdelen@mersin.edu.tr

A. Y1lmaz

e-mail: yilmazarda@hotmail.com

N. G. Bilgin

e-mail: nurselbilgin@gmail.com

A. Özge $(\bowtie)$

Mersin Üniversitesi Tıp Fakültesi Nöroloji AD, Mersin, Turkey

e-mail: aynurozge@gmail.com $(p=0.006)$. Second, having any triggers caused 1.440 -fold increase in the primary headache risk $(p=0.001)$. Third, having associated co-morbid medical disorders caused 4.643-fold increase in the secondary headache risk $(p<0.001)$. It was concluded that the presence of comorbidity, the patient's age, the existence of trigger and relaxing factors, the pain in other body parts that accompanies headache and the quality of pain in terms of location and duration were all important clues for physicians in making an accurate differentiation between primary and secondary headaches.

Keywords Emergency · Headache - Primary headaches . Secondary headaches - Classification and regression tree . Differential diagnosis

\section{Introduction}

The occurrence of headache as the chief complaint on admission to an emergency room (ER) is between $1 \%$ and $16 \%$ [1-4]. Headache is a symptom related to many disorders, ranging from benign to more serious diseases. Although most headaches are benign, it is the responsibility of ER and primary healthcare physicians to obtain a detailed history and a careful examination to rule out other causes of pain with significant morbid or fatal outcome. Therefore, the ER practitioner must recognize symptoms and characteristics of headache in order to distinguish who simply needs reassurance and analgesia and who needs prompt diagnosis and interventions [4-7].

In order to obtain a correct diagnostic and therapeutic pathway and ask for targetted specialist consultation, the ER practitioner must be familiar with the various categories of headache. The first great subdivision can be made between 
primary and secondary headaches due to a specific reason [7]. Classifying headaches as primary or secondary can also facilitate evaluation and management [8]. Primary headaches have no structural or metabolic cause, while secondary headaches are caused by an underlying pathologic or metabolic process. Migraine, tension-type, cluster, and analgesicrebound headaches are all primary headache disorders. Secondary headaches are caused by conditions such as increased intracranial pressure, pseudotumour, subdural haematomas, ischaemic as well as haemorrhagic strokes, hypertension, meningitis, temporal arteritis, and brain tumours. The majority of patients presenting to ER practitioners complaining of headache have primary headaches, and approximately only $10 \%$ of them have a secondary form $[5,9,10]$. In most cases, neurological examination was normal. A complete headache history contributes most to the diagnostic process. History should include information about headache onset, pain intensity and character of the pain, presence of aura, associated autonomic symptoms, and trigger factors [10, 11]. Cortelli et al. [4] stated that the first step in evaluating headache patients accessing ERs involves exclusion of secondary headaches that reflect an underlying life-threatening condition. "Red flags" for secondary disorders include sudden onset of headache, new onset of headache with aura, onset of headache after 50 years of age, increased frequency or severity of headache, new onset of headache with an underlying medical condition, headache with concomitant systemic illness $[2,8]$. The detailed diagnostic criteria and clinical variabilities for headaches are well known by neurologists, but there is a need to obtain a practical guide to the differential diagnosis of headaches for ER practitioners and primary healthcare physicians.

Some previous reports pointed out the importance of the dissemination of the International Headache Society (IHS) diagnostic criteria among primary healthcare physicians in order to avoid the return of patients or their referral to more specialized ERs [5, 6]. The correct diagnosis of headache disorders in ER is also very important for developing early management strategies and for determining optimal ER activities. For these reasons, there is need to obtain an easy guide to evaluate headaches for ER practitioners. We performed this multidisciplinary prospective clinical based study in order to determine demographic and clinical clues for differential diagnosis of primary and secondary headache disorders and also to obtain a classification plot for ER practitioners.

\section{Materials and methods}

We conducted a prospective study of consecutive adult (age $>15$ years) patients presenting with a chief complaint of nontraumatic headache to the ER of Mersin University
Medical Faculty Hospital from March 2006 to September 2006. The hospital is an academic hospital and it receives patients from an extensive region. The ER sees approximately 20,000 adult cases annually. Written informed consent was obtained from all patients.

All of the consecutive patients older than 15 years of age were included in the study on the basis of a chief complaint of nontraumatic headache. Patients were excluded from the study if headache was not the chief complaint on admission but detected while obtaining medical history. Other patients excluded were those with headaches resulting from trauma. Patients unwilling or unable to provide informed consent were also excluded from the study.

ER practitioners were asked to fill out a patient questionnaire for headache sufferers after obtaining an informed consent from the patient or her/his family member. This questionnaire was developed utilizing "The second edition of The International Classification of Headache Disorders (ICHD-2)" criteria, including questions related to the information about demographic and clinical data [12]. By this way, informative data about frequency, severity, quality, localization and duration of headache, its timing, symptoms that accompanied it, the pain from other body regions, the presence of relevant co-morbidities, previous diseases, family history of headache disorders, any medication taken before admission, any drugs the patient was using, diagnostic tests ordered, therapies employed and response to treatments were obtained.

After ER practitioner's evaluations, the neurologist in charge was also asked to examine the patients to indicate diagnostic tests and to make the headache diagnosis according to the ICHD- 2 criteria. Necessary laboratory and radiography studies and required consultations were performed to establish a definitive diagnosis. All steps of the neurological evaluations were supervised by the headache specialist (A.Ö.), and definite diagnoses of the subjects were also made by her. After ER setting process, all headache patients, except for secondary headaches that relieved after solving secondary causes, were referred to our outpatient headache centre. It is one of the sections of neurology department. Most of the patients referred have primary headaches. Minimum follow-up duration of headaches in this centre is 6 months. The diagnosis was made mostly in the ER. But in some headache patients, we needed a followup period in order to make distinct diagnosis. The final diagnoses were controlled by assessing findings that obtained both from the ER and the headache centre.

The data were processed and analysed using the statistical package SPSS-11.0 for Windows. The results of descriptive analyses were tested and found to show equal distribution. Mean and standard deviations of data were calculated. Continuous data were compared using independent $t$ and Mann-Whitney $U$ tests. Categorical data 
were compared using the $\chi 2$ or Fisher exact tests. Since the study is a prospective clinical based study, we used relative risk (RR) as a coefficient for the risk factors; these relative risks were estimated from univariate cross tabs analysis. Post hoc power analysis was performed for the variables found to be statistically significant by univariate analysis to determine whether the sample size was large enough to be statistically valid. The significant variables were also included in a multivariate binary logistic regression analysis model to determine most predictive factors for headache. Significant differences (two-tailed $p$ ) less than 0.05 were regarded as significant.

We used Statistica ${ }^{\circledR} 6.0$ (STATISTICA, 1984-2002) software for the classification and regression tree (CRT). At the initial stage, the building of CRT began with a root node, which contained all of the subjects; then, a series of yes/no questions generated descendant nodes. These nodes were more homogenous than the root node and these terminal homogenous nodes were evaluated.

Totally, 174 patients were included in this analysis and named as "learning sample". As a dependent variable, the diagnosis of primary headache was coded as 1 (yes) and 0 (no $=$ secondary headache). The variables which possibly affect the headache diagnosis were accepted as independent variables. It was composed of 12 categorical variables and one ordered variable, totally 13 clinical variables. Tenfold cross validation was used as an error estimation method. After the maximum tree value had been obtained, the pruning process began in order to obtain optimum tree set, using the cost-complexity setting method, providing the balance between probability of misclassification calculation from risk matrix and tree complexity. After obtaining the optimum tree, the results of this method have been evaluated.

\section{Results}

While obtaining medical history from the patients, careful attention was given to the reasons for ER attendance in order not to miss any patient with headache as the chief complaint. All of the consecutive patients who met inclusion criteria were enrolled in the study after obtaining informed consent. Overall, the final number of our study subjects was 174 , with a mean age of $40.05 \pm 13.94$ years (range, 16-78 years). Females were found to be predominant $(76.4 \%, 133$ patients). Demographic features of the study groups are shown in Table 1 . The patients were diagnosed as basically primary $(n=127,72.9 \%)$ and secondary $(n=47,27.1 \%)$ headache disorders according to ICHD-2 criteria.

Of all headache subjects included in our study 12 underwent computerize tomography (CT). Subarachnoid haemorrhage was detected in three of them, while others had normal CT results. Of those that underwent CT, five patients were diagnosed as having primary headaches (all migraine), seven patients were diagnosed as having secondary headaches (three subarachnoid haemorrhage, three hypertension, and one panic disorder). All of the subjects with negative CT and suspected history of subarachnoid haemorrhage underwent lumbar puncture (LP) but the final diagnosis did not change. Of subjects coded with 6.3.1 (headache attributed to saccular aneurysm), 6.4 (headache attributed to arteritis), 7.4 (headache attributed to intracranial neoplasm), 7.6 (headache attributed to epileptic seizure) and 9.2 (headache attributed to systemic infection) were hospitalised (totally eight subjects, $4.6 \%$ ) for further medical investigations and treatment (see Table 2).

Diagnoses of headache disorders according to ICHD-2 criteria are summarized in Table 2. As shown in this table, primary headache disorders were the most common cause of ER admissions and among them migraine was the most frequent type, followed by infrequent episodic tension-type headache (ETTH). Among the secondary type headache disorders, headache attributed to arterial hypertension was the most common cause, followed by headache attributed to psychiatric disorders.

Detailed descriptions and the results of the structured interview of subjects showed that unilateral location of headache, having a trigger factor for headache and having some associated features during attacks had statistically significant effects on primary headache diagnosis (see Table 3).

Univariate analysis for the study variables was conducted by using cross tabs. The results obtained from the univariate analysis showed three important results. First, unilateral pain location caused 1.431-fold increase in the primary headache risk (95\%CI: $1.291-1.587, p=0.006$ ). Second, having any trigger factor caused 1.440-fold increase in the primary headache risk $(95 \% \mathrm{CI}$ : 1.113 1.863, $p=0.001$ ). Third, having associated co-morbid medical disorder caused 4.643-fold increase in the secondary headache risk (95\% CI: 2.203-9.783, $p<0.001$ ). Before clinical interpretation of the above mentioned univariate analysis results, post hoc power analysis was performed to determine whether the sample size was large enough for the findings to be statistically valid. The power values for the parameters including the presence of unilateral pain location, trigger factors, and co-morbid medical disorders were found to be as 76.3, 87.06, and $99.89 \%$, respectively. Variables found to be statistically significant by univariate analysis were also included in a multivariate binary logistic regression analysis model to determine which variables were the most important in differentiating primary headaches from secondary ones. Odds ratio could not be calculated for the unilateral pain 
Table 1 Demographic features of patients

\begin{tabular}{|c|c|c|c|c|}
\hline Variables & $\begin{array}{l}\text { Primary headache } \\
(n=127)\end{array}$ & $\begin{array}{l}\text { Secondary headache } \\
(n=47)\end{array}$ & $p$ & $\chi^{2 / t}$ score \\
\hline Age (year) (mean $\pm \mathrm{SD})$ & $38.8 \pm 11.8$ & $48.6 \pm 15.5$ & 0.000 & -5.33 \\
\hline Gender (female/male) (\%) & $96(75.6) / 31(24.4)$ & $37(78.7) / 10(21.3)$ & 0.665 & - \\
\hline Marital status $(n)(\%)$ & & & 0.034 & 6.78 \\
\hline Married & $96(75.6 \%)$ & $42(89.3 \%)$ & & \\
\hline Single & $30(23.6 \%)$ & $3(6.5 \%)$ & & \\
\hline Divorced & $1(0.8 \%)$ & $2(4.2 \%)$ & & \\
\hline Education time (years) (mean \pm SD) & $9.5 \pm 4.0$ & $7.6 \pm 4.0$ & 0.007 & 2.72 \\
\hline Employment status $(n)(\%)$ & & & 0.013 & - \\
\hline Employed & $59(46.5 \%)$ & $12(23.9 \%)$ & & \\
\hline Unemployed & $68(53.5 \%)$ & $35(76.1 \%)$ & & \\
\hline First attendance $(n)(\%)$ & $80(63.0 \%)$ & $28(59.6 \%)$ & 0.68 & - \\
\hline Other medical conditions $(n)(\%)$ & & & 0.000 & 54.31 \\
\hline None & $71(55.9 \%)$ & $7(14.9 \%)$ & & \\
\hline Hypertension & $8(6.3 \%)$ & $12(25.5 \%)$ & & \\
\hline Endocrinologic disorders & $2(1.6 \%)$ & $4(8.5 \%)$ & & \\
\hline Coronary artery disorders & 0 & $1(2.1 \%)$ & & \\
\hline Hematological disorders & $1(0.8 \%)$ & $4(8.5 \%)$ & & \\
\hline Depressive disorders & $13(10.2 \%)$ & $4(8.5 \%)$ & & \\
\hline Anxiety disorders & $6(4.7 \%)$ & $6(12.8 \%)$ & & \\
\hline Allergic disorders & $3(2.4 \%)$ & $2(4.3 \%)$ & & \\
\hline Snoring & $9(7.1 \%)$ & 0 & & \\
\hline Epilepsy & $2(1.6 \%)$ & $2(4.3 \%)$ & & \\
\hline Others & $12(9.4 \%)$ & $5(10.6 \%)$ & & \\
\hline Triggers for headache $(n)(\%)$ & & & 0.015 & 14.15 \\
\hline None & $29(22.9 \%)$ & $23(48.9 \%)$ & & \\
\hline Stress & $73(57.5 \%)$ & $19(40.4 \%)$ & & \\
\hline Occupation & $5(3.9 \%)$ & $1(2.1 \%)$ & & \\
\hline Others & $20(15.7 \%)$ & $4(8.6 \%)$ & & \\
\hline Family history of headache $(n)(\%)$ & $58(45.6 \%)$ & $14(29.7 \%)$ & 0.059 & - \\
\hline
\end{tabular}

SD Standard deviation

location parameter, because all of the unilateral headaches were in primary headache group. As a result, odds ratios were calculated for the parameters including the presence of trigger factors and co-morbid medical disorders and found to be as 3.755 (CI: 1.710-8.249, $p=0.001)$ and, 8.014 (CI: 3.218-19.956, $p<0.001$ ) respectively.

\section{Classification and regression tree}

In order to obtain the optimum tree, cross validation and resubstitution cost values were calculated as $0.323 \pm 0.0405$ and 0.1118 , respectively. These values were the estimations of error variance of the tree. Therefore, it can be concluded that the terminal nodes were homogeneous groups. The complexity parameter was 0.000 .
The CRT results provided us some clinical clues to the differential diagnosis of headaches in ER settings (see Fig. 1). First, a clinician should perform a complete medical evaluation in order to indicate or rule out the presence of co-morbid medical disorders, as comorbidity was found to be the main factor in accurate differentiation of primary headaches from secondary ones. If comorbidity is present, then the patient's age, the location and duration of pain, the presence of accompanying neck or back pain, and the existence of trigger factors will become helpful diagnostic clues in the differentiation process of headache disorders. Furthermore, in headache patients without comorbidity, particular attention should be given to the presence of accompanying neck or low back pain, the patient's age, the existence of relaxing factors and the duration of pain. The key features obtained from CRT results could be explained as follows: 
Table 2 Diagnosis of headache disorders

\begin{tabular}{|c|c|c|c|c|}
\hline & $\operatorname{Code}^{\mathrm{a}}$ & Definition & Frequency & Percent \\
\hline \multirow[t]{9}{*}{ Primary headache disorders } & 1.1 & Migraine without aura & 56 & 32.2 \\
\hline & 1.3 & Migraine with aura & 11 & 6.3 \\
\hline & 1.5 & Complications of migraine & 2 & 1.1 \\
\hline & 1.6 .1 & Probable migraine without aura & 13 & 7.5 \\
\hline & 2.1 & Infrequent episodic tension & 26 & 14.9 \\
\hline & 2.2 & Frequent episodic tension & 5 & 2.9 \\
\hline & 2.3 & Chronic tension & 5 & 2.9 \\
\hline & 2.4 & Probable tension & 3 & 1.7 \\
\hline & 4.1 & Primary stabbing headache & 3 & 1.7 \\
\hline \multirow[t]{12}{*}{ Secondary headache disorders } & 6.3 .1 & Headache attributed to saccular aneurysm ${ }^{\mathrm{b}}$ & 3 & 1.7 \\
\hline & 6.4 & Headache attributed to arteritis ${ }^{\mathrm{b}}$ & 1 & 0.6 \\
\hline & 7.4 & Headache attributed to intracranial neoplasm ${ }^{\mathrm{b}}$ & 2 & 1.1 \\
\hline & 7.6 & Headache attributed to epileptic seizure & 1 & 0.6 \\
\hline & 9.2 & Headache attributed to systemic infection & 1 & 0.6 \\
\hline & 10.3 & Headache attributed to arterial hypertension & 22 & 12.6 \\
\hline & 10.4 & Headache attributed to hypothyroidism & 1 & 0.6 \\
\hline & 11.2 & Headache attributed to disorder of neck & 1 & 0.6 \\
\hline & 11.5 & Headache attributed to rhinosinusitis & 3 & 1.7 \\
\hline & 11.7 & Headache or facial pain attributed to temporomandibular joint disorder & 1 & 0.6 \\
\hline & 12.1 & Headache attributed to somatisation disorders & 11 & 6.3 \\
\hline & 14.1 & Headache not elsewhere classified & 3 & 1.7 \\
\hline
\end{tabular}

${ }^{a}$ Refer to the second edition of The International Classification of Headache Disorders (ICHD-2) codes. ${ }^{11}$

b The subjects with headache requiring emergency workup and management

The patient has an increased risk of primary headache under following conditions;

- Neither comorbidity nor neck-low back pain is present.

- If comorbidity is absent, age is between 20 and 40 years, and neck-low back pain accompanies the headache.

- If comorbidity is absent, age is between 40 and 60 years, neck-low back pain accompanies the headache, relaxing factor is present, and headache lasts less than $24 \mathrm{~h}$.

- If comorbidity is present, age is between 20 and 50 years, and headache is unilateral.

- If comorbidity is present, age is between 20 and 50 years, and headache is diffuse without trigger factors.

- If comorbidity is present, age is between 20 and 50 years, headache is diffuse with trigger factors, and lasts more than $24 \mathrm{~h}$.

- If comorbidity is present, age is between 20 and 50 years, headache is diffuse with trigger factors, but lasts less than $24 \mathrm{~h}$, while no neck pain accompanies it.
- If comorbidity is present, age is between 50 and 60 years, and neck-back pain accompanies the headache, while no trigger factor is present.

- If comorbidity is present, age is over 50 years, and headache is unilateral, but no neck-back pain accompanies it.

The patient has an increased risk of secondary headache under following conditions;

- If comorbidity is absent, age is over 40 years, and neck-low back pain accompanies the headache, while no relaxing factor is present.

- If comorbidity is absent, age is over 60 years, and neck-low back pain accompanies the headache, while relaxing factor is present.

- If comorbidity is absent, age is between 40 and 60 years, neck-low back pain accompanies the headache, relaxing factor is present, and headache lasts more than $24 \mathrm{~h}$.

- If comorbidity is present, age is between 20 and 50 years, neck pain accompanies diffuse headache, trigger factor is present, and headache lasts less than $24 \mathrm{~h}$. 
Table 3 Headache features of study subjects

\begin{tabular}{|c|c|c|c|}
\hline Variables & Primary headache $(n=127)$ & Secondary headache $(n=47)$ & $p$ \\
\hline Duration of headache (months) (mean \pm SD) & $41.6 \pm 52.4$ & $47.5 \pm 73.8$ & 0.378 \\
\hline Duration of attacks $(\mathrm{h})($ mean $\pm \mathrm{SD})$ & $15.0 \pm 20.0$ & $12.5 \pm 17.4$ & 0.267 \\
\hline Frequency of attacks $(n)(\%)$ & & & 0.231 \\
\hline First time $(n=19)$ & $11(8.7 \%)$ & $8(17.0 \%)$ & \\
\hline Less than one per month $(n=76)$ & $61(48.1 \%)$ & $15(31.9 \%)$ & \\
\hline $1-5$ days in a month $(n=21)$ & $15(11.8 \%)$ & $6(12.8 \%)$ & \\
\hline $10-15$ days in a month $(n=23)$ & $19(15.0 \%)$ & $4(8.5 \%)$ & \\
\hline More than 15 days in a month $(n=7)$ & $4(3.1 \%)$ & $3(6.4 \%)$ & \\
\hline Everyday $(n=28)$ & $17(13.4 \%)$ & $11(23.4 \%)$ & \\
\hline Severity of attacks $(n)(\%)$ & & & 0.534 \\
\hline Mild $(n=3)$ & $2(1.6 \%)$ & $1(2.1 \%)$ & \\
\hline Moderate $(n=26)$ & $20(15.7 \%)$ & $6(12.8 \%)$ & \\
\hline Severe $(n=64)$ & $50(39.4 \%)$ & $14(29.8 \%)$ & \\
\hline Very severe $(n=81)$ & $55(43.3 \%)$ & $26(55.3 \%)$ & \\
\hline Location of headache $(n)(\%)$ & & & 0.04 \\
\hline Unilateral $(n=18)$ & $18(14.2 \%)$ & 0 & \\
\hline Bilateral $(n=85)$ & $59(46.5 \%)$ & $26(55.3 \%)$ & \\
\hline Secondary generalised $(n=6)$ & $5(3.9 \%)$ & $1(2.1 \%)$ & \\
\hline Calvarial $(n=17)$ & $10(7.9 \%)$ & $7(14.9 \%)$ & \\
\hline Temples $(n=14)$ & $12(9.4 \%)$ & $2(4.3 \%)$ & \\
\hline Frontal $(n=16)$ & $13(10.2 \%)$ & $3(6.4 \%)$ & \\
\hline Suboccipital $(n=18)$ & $10(7.9 \%)$ & $8(17 \%)$ & \\
\hline Quality of headache $(n)(\%)$ & & & 0.829 \\
\hline Throbbing $(n=117)$ & $87(68.5 \%)$ & $30(63.8 \%)$ & \\
\hline Pressing $(n=51)$ & $36(28.4 \%)$ & $15(31.8 \%)$ & \\
\hline Stabbing $(n=6)$ & $4(3.1 \%)$ & $2(4.3 \%)$ & \\
\hline Triggers $(n)(\%)$ & & & 0.015 \\
\hline None $(n=52)$ & $29(22.8 \%)$ & $23(48.9 \%)$ & \\
\hline Stress $(n=92)$ & $73(57.5 \%)$ & $19(40.4 \%)$ & \\
\hline Others $(n=30)$ & $25(19.7 \%)$ & $5(10.7 \%)$ & \\
\hline Associated features $(n)(\%)$ & & & 0.048 \\
\hline None $(n=30)$ & $22(17.3 \%)$ & $8(17.0 \%)$ & \\
\hline Nausea $(n=22)$ & $15(11.8 \%)$ & $7(14.9 \%)$ & \\
\hline Vomiting $(n=2)$ & $2(1.6 \%)$ & 0 & \\
\hline Photophobia $(n=4)$ & $3(2.4 \%)$ & $1(2.1 \%)$ & \\
\hline Phonophobia $(n=5)$ & $4(3.1 \%)$ & $1(2.1 \%)$ & \\
\hline More than two associated features $(n=101)$ & $78(61.4 \%)$ & $23(48.9 \%)$ & \\
\hline Others $(n=10)$ & $3(2.4 \%)$ & $7(14.9 \%)$ & \\
\hline Timing of headache $(n)(\%)$ & & & 0.406 \\
\hline None $(n=112)$ & $77(60.6 \%)$ & $35(74.5 \%)$ & \\
\hline Mornings $(n=21)$ & $17(13.4 \%)$ & $4(8.5 \%)$ & \\
\hline Afternoons $(n=22)$ & $18(14.2 \%)$ & $4(8.5 \%)$ & \\
\hline Nights $(n=19)$ & $15(11.8 \%)$ & $4(8.5 \%)$ & \\
\hline
\end{tabular}

- If comorbidity is present, age is over 50 years, neckback pain accompanies the headache, and trigger factor is present.
- If comorbidity is present, age is over 50 years, and headache is diffuse, but no neck-back pain accompanies it. 
Fig. 1 Classification and regression tree of main headache disorders

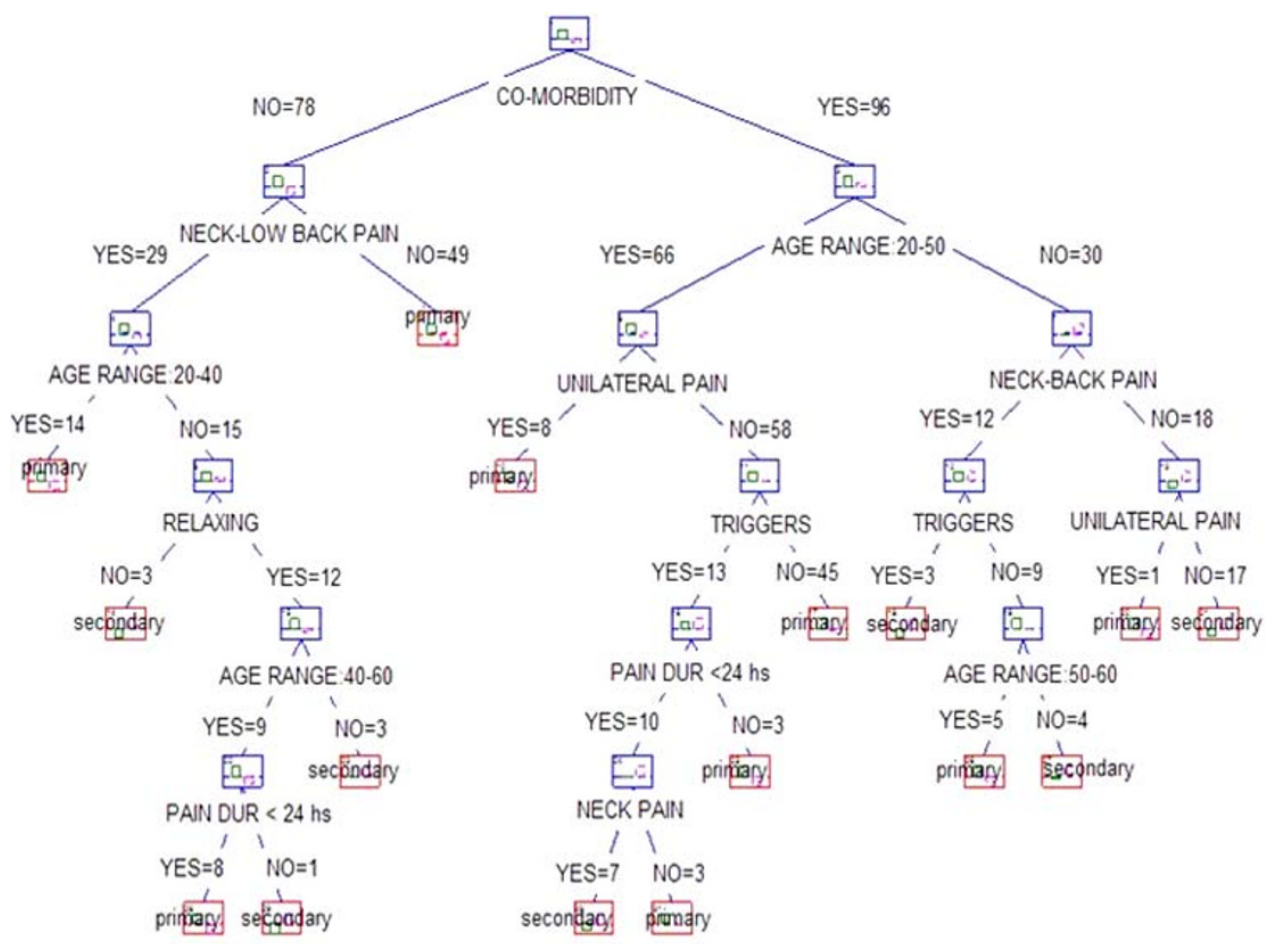

- If comorbidity is present, age is over 60 years, and neck pain accompanies the headache, while no trigger factor is present.

However, the most important feature among the key clues explained above is that if comorbidity is present, age is over 50 years, and trigger factor is present, then the patient has a 9.33 -fold increased risk of secondary headache $(p=0.032)$.

\section{Discussion}

Clinicians on duty at busy ERs are frequently faced with difficulties in assessing patients with headache. Bigal et al. [13] reported that the primary care for headache was unsatisfactory in the ER; therefore, more educated practitioners and new easy guidelines were needed for the accurate diagnosis of headache in the ER settings. Landtblom et al. [14] also stressed the importance of the rapid differential diagnosis process in ER settings.

For the past 15 years, the diagnostic criteria of the IHS have been the accepted standards. The ICHD-2 reflects our improved understanding of some disorders and the identification of new disorders, and it also provides a subgroup analysis of primary headaches and detailed description for headache attributed to psychiatric disorders [12]. Wellestablished criteria are available for headache and in most cases a diagnosis can be made without the use of laboratory tests, but these criteria cannot be routinely employed in busy ERs [6, 13]. Furthermore, classical features of the main headache types are generally well known to the neurologists, but ER practitioners have little training in accurate headache diagnosis, and thus most headaches may be misdiagnosed by them in daily practice $[6,7,12,15]$. As a result, an easy algorithm to guide the classification of headaches according to the IHS criteria is needed to help ER physicians in making rapid and accurate differentiation of primary headaches from secondary ones $[8,16]$.

An important paper creating four scenarios for the ER-based differential diagnosis of headache disorders, using databases and guidelines, was reported by Cortelli et al. [4]. Their suggestions represented a compromise between accuracy and clinical pragmatisms for the ERbased headache differential diagnosis. Based on this report we designed the present study to provide positive contributions to their suggestions, and used CRT for the same purposes. To our knowledge no guidelines similar to ours have been published to date.

Our CRT-based results showed that the presence of comorbidity, the patient's age, the existence of trigger and relaxing factors, the pain of the other body parts that accompanies headache (neck, back and low back pain) and the quality of pain in terms of location and duration were all important clues for ER physicians in making an accurate differentiation between primary headaches and those from secondary causes. Obtaining such a complete headache and medical history with elements mentioned above have also been reported as the most important parts of the initial diagnostic evaluation $[7,10,17]$. 
In our study, comorbidity was found to be the main factor in accurate differentiation of primary headaches from secondary ones. The importance of obtaining information about comorbidity in headache patients has been stressed by many authors, but it has not been used as a classifier in the differential diagnostic process of headaches $[5,8,17]$.

Emergency physicians should also pay attention to the patient's age in the differential diagnostic process of headaches. Headaches beginning under the third decade of life are frequently migraines. Those beginning later may have an organic aetiology [16]. Bigal et al. [5] reported that there was a sharp predominance of primary headaches, whose frequency increased up to the 40-50 year age range and declined thereafter. Considering that the majority of patients who present to the ERs with acute primary headache have migraine, although most do not receive this diagnosis, the age of migraine patients becomes an important determinator in the differential diagnostic process [6]. Migraine predominantly affects women and reaches its peak between 25 and 55 years of age, while more than $90 \%$ of migraineurs experience their first attack before 40 years [15]. Similar to migraine, the age at onset of cluster headache is generally less than 30 years $(15,18)$. Secondary headaches are more frequent in patients over 50 years; approximately $65 \%$ of $\mathrm{SAH}$ occur during the fourth to sixth decade [15]. However, the limited data mentioned above are inadequate to explain the guiding role of the patient's age in the differential diagnostic process of headaches. In our study we suggested to consider primary headaches in young patients (between 20 and 50 years of age) with comorbidity whenever headache is unilateral and also in young patients (between 20 and 40 years of age) without comorbidity, if neck-low back pain accompanies it. We also suggested that clinicians should suspect secondary headaches in patients over 50 years of age whenever neck-back pain and trigger factors are present, or in patients over 60 whenever neck-back pain is present but trigger factor is absent. If the headache is diffuse in elder patients without neck-back pain, secondary headaches should also be considered.

Pain characteristics are other important elements in the evaluation of headache patients. A comprehensive history of patients presenting in ER with headache should include questions about quality, location and duration of the pain as well as about conditions which relieve or exacerbate it [15]. Among the restricted reports in this area, Verslegers et al. [18] described cluster headache as mostly severe and mainly unilateral pain, which is located outside the orbitotemporal region and often lasts more than $180 \mathrm{~min}$. They reported that the initial pain may be located in the neck and the neck pain can overshadow all other symptoms of cluster headache. Gardinali et al. [15] stated that pain is invariably unilateral in cluster headache and in the majority of migraine attacks. These statements are in good agreement with our findings. Our results also suggested considering primary headaches both in young and older patients with comorbidity whenever headache is unilateral, but to consider secondary headaches in older individuals if the headache is diffuse and neck-back pain does not accompany it. Some reports related to the pain duration in headache patients have indicated that migraine lasts 4-72 h with a median frequency of 1.5 per month and cluster headache lasts 15-180 min with attacks recurring from one every other day to eight per day, while SAH headache lasts from at least $1 \mathrm{~h}$ to days to weeks [15]. Our study made positive contributions to these reports, as our findings suggested that primary headaches should be considered if headache lasts less than $24 \mathrm{~h}$ in older patients without comorbidity who have neck-low back pain and relaxing factor, while duration of more than $24 \mathrm{~h}$ in those patients suggests secondary headaches.

Bigal et al. [5] reported that the most frequent aetiologies of secondary headache disorders were fewer, acute arterial hypertension, and sinusitis. But hypertension could also be one of the factors leading to exacerbation of the frequency and severity of attacks, both in migraine and tension-type headache, and the evidence supporting a connection between chronic hypertension and headache is scant $[15,19]$. As a result, in our study hypertension was not found to be a major factor in the differentiation of secondary headaches from primary ones.

The present study has some limitations. First of all, the study was designed as a hospital-based and a single-centre survey. The rest of ER attendees whose chief complaint was not headache, in other words, in whom headache was only a symptom accompanying any other chief complaint, had to be ignored. And the results could not be accepted as a population-based representation. Second, this was a tertiary university hospital, and our descriptive results may not be necessarily applicable to different hospital settings. Third, because of the sample size, subgroups apart from primary and secondary headache disorders could not be analysed separately. Therefore, a more detailed guideline including these subgroups could not be made.

This study has also several implications. First, in the present study, which was designed in order to obtain a classification plot, required data had been recorded by the ER specialists, not by the subjects themselves. Second, to the best of our knowledge, this is the first study in making differential diagnosis by using CRT as a new method for statistics. Third, in our study we used relative risk estimation and CRT methods together in order to obtain important variables for the differential diagnosis process and relative risk coefficient to achieve the best results. For these reasons, our results can be projected to more 
comprehensive clinical-based studies in order to obtain headache management algorithms.

It was concluded that the presence of comorbidity, the patient's age, the existence of trigger and relaxing factors, the pain in other body parts that accompanies headache (neck, back and low back pain) and the quality of pain in terms of location and duration were all important clues for ER physicians in making an accurate differentiation between primary headaches and those from secondary causes.

\section{References}

1. Morgenstern LB, Huber JC, Luna-Gonzales H, Saldin KR, Grotta JC, Shaw SG, Knudson L, Frankowski RF (2001) Headache in the emergency department. Headache 41:537-541

2. Agostoni E, Santoro P, Frigerio R, Frigo M, Beghi E, Ferrarese C (2004) Management of headache in emergency room. Neurol Sci 25(Suppl 3):187-189

3. Carroll C, Zajicek J (2004) Provision of 24 hour acute neurology care by neurologists: manpower requirements in the UK. J Neurol Neurosurg Psychiatry 75:406-409

4. Cortelli P, Cevoli S, Nonino F, Baronciani D, Magrini N, Re G, De Berti G, Manzoni GC, Querzani P, Vandelli A (2004) Evidence-based diagnosis of nontraumatic headache in the emergency department: a consensus statement on four clinical scenarios. Headache 44:587-595

5. Bigal ME, Bordini CA, Speciali JG (2000) Etiology and distribution of headaches in two Brazilian primary care units. Headache 40:241-247

6. Blumenthal HJ, Weisz MA, Kelly KM, Mayer RL, Blonsky J (2003) Treatment of primary headache in the emergency department. Headache 43:1026-1031
7. Gaini SM, Fiori L, Cesana C, Vergani F (2004) The headache in the Emergency Department. Neurol Sci 25(Suppl 3):196-201

8. Clinch CR (2001) Evaluation of acute headaches in adults. Am Fam Physician 63:685-692

9. Peters KS (2004) Secondary headache and head pain emergencies. Prim Care 31:381-393

10. Marks DR, Rapoport AM (1997) Practical evaluation and diagnosis of headache. Semin Neurol 17:307-312

11. Locker T, Mason S, Rigby A (2004) Headache management-are we doing enough? An observational study of patients presenting with headache to the emergency department. Emerg Med J 21:327-332

12. Headache Classification Subcommittee of the International Headache Society (2004) The international classification of headache disorders, 2nd edn. Cephalalgia 24(Suppl 1):9-160

13. Bigal M, Bordini CA, Speciali JG (2000) Headache in an emergency room in Brazil. Sao Paulo Med J 118:58-62

14. Landtblom AM, Fridriksson S, Boivie J, Hillman J, Johansson G, Johansson I (2002) Sudden onset headache: a prospective study of features, incidence and causes. Cephalalgia 22:354-360

15. Gardinali M, Bernareggi M, Magni S (2004) Headaches in the emergency department. Neurol Sci 25(Supply 3):192-195

16. Svenson J, Cowen D, Rogers A (1997) Headache in the emergency department: importance of history in identifying secondary etiologies. J Emerg Med 15:617-621

17. Maizels M (2004) The patient with daily headaches. Am Fam Physician 70:2299-2306

18. Verslegers WR, Pickut BA, De Deyn PP (2006) Paroxysmal neuralgic upper cervical pain attacks: the lower syndrome of cluster headache. Clin Neurol Neurosurg 108:737-743

19. Pietrini U, De Luca M, De Santis G (2005) Hypertension in headache patients? A clinical study. Acta Neurol Scand 112:259-264 